SUBANTRAL AUGMENTATION WITH A 1/1 ALLOPLASTIC AND AUTOLOGOUS MATERIAL MIXTURE. HISTOLOGICAL CHARACTERISTICS AND DIMENSIONAL STABILITY OF THE NEWLY FORMED BONE

\title{
FLORIN ONIȘOR-GLIGOR ${ }^{1}$, MIHAI JUNCAR ${ }^{1}$, GRIGORE BĂCIUȚ ${ }^{1}$, SIMION BRAN ${ }^{2}$, RALUCA-IULIA JUNCAR ${ }^{3}$, DINU-IULIU DUMITRAȘCU ${ }^{4}$, MIHAELA-FELICIA BĂCIUT⿻ ${ }^{2}$
}

\author{
${ }^{1}$ Department of Oral and Maxillofacial Surgery, Iuliu Hatieganu University of \\ Medicine and Pharmacy, Cluj-Napoca, Romania \\ ${ }^{2}$ Department of Maxillofacial Implantology and Surgery, Iuliu Hatieganu \\ University of Medicine and Pharmacy, Cluj-Napoca, Romania \\ ${ }^{3}$ Department of Prosthetics, Iuliu Hatieganu University of Medicine and \\ Pharmacy, Cluj-Napoca, Romania \\ ${ }^{4}$ Department of Anatomy and Embryology, Iuliu Hatieganu University of \\ Medicine and Pharmacy, Cluj-Napoca, Romania
}

\begin{abstract}
Background and aim. The maxillary sinus augmentation procedure is the most frequent method used to prepare the posterior maxillary area, with bone deficit, for the placement of dental implants. For this purpose, several types of materials have been used as bone grafts, each of these materials having a number of advantages and disadvantages. The present study is aimed at evaluating the histological and volumetric properties of an alloplastic and autologous material mixture.

Patients and methods. The study included 7 selected patients who underwent subantral bone augmentation with a 1/1 autologous and alloplastic material mixture. Subsequently, at the time of dental implant placement, biological samples were taken from the bone augmentation area and were histologically analyzed. The subantral bone resorption rate was also evaluated in parallel.

Results. Histological examination evidenced the presence of residual amorphous material in the bone augmentation area. The mean bone resorption rate was $15.15 \%$. The presence of a high bone resorption rate was correlated with the presence of a smaller residual amorphous material amount in the subantral bone augmentation area.

Conclusions. The association of autologous and alloplastic material for subantral bone augmentation improves the characteristics of the two material types.
\end{abstract}

Keywords: sinus lift, alloplastic graft, autologous graft, bone substitute.

\section{Introduction}

Dental implants are currently the optimal method for the functional and aesthetic restoration of edentulous areas both at maxillary and mandibular level [1]. However, a number of local problems may frequently limit the use

Manuscript received: 04.01.2015

Accepted: 19.01.2015

Address for correspondence: mihaijuncar@gmail.com of dental implants, and one of the regions posing the most problems is the posterior maxillary area. The main inconvenient of this area is the insufficient bone available as a result of post-extraction bone resorption or maxillary sinus pneumatization $[2,3]$. Several surgical methods have been designed and applied in medical practice to overcome this limitation, such as special implants, subantral bone augmentation, onlay bone augmentation, etc. Of these 
methods, the most widely used and accepted in current medical practice is subantral bone augmentation, known as sinus lift [4].

The sinus lift procedure is a method by which a material is placed in the sinus floor, in a space created by the elevation of the sinus membrane from the bone support represented by the maxillary sinus floor [4]. This bone augmentation is performed using several methods. One of the most frequently used methods is that described by Tatum [3], which involves the approach of the maxillary sinus through a window opened in the lateral sinus wall. Four types of materials have been proposed and employed for bone augmentation: autogenous bone, allogenic bone, xenogenic bone, and alloplastic bone [1,2]. Of these, autogenous bone is considered to have the best properties as a bone augmentation material $[1,5,6]$. Although autologous bone undoubtedly has osteoinductive characteristics, its high resorption rate after bone augmentation has been evidenced, which is why some authors have recommended its association with xenogenic bone or alloplastic bone, which have a slower resorption rate $[7,8]$.

The aim of this study is to prospectively evaluate the quality of bone tissue obtained after the performance of sinus lift with a 1/1 mixture of autogenous and alloplastic material. At the same time, the resorption rate of this type of material 6-8 months after bone augmentation is assessed.

\section{Patients and methods}

The study included 7 patients selected from the patients treated by the research team members, who in the period December 2013 - March 2014 requested implant placement in the posterior maxillary region and needed subantral bone augmentation. All patients included in the study voluntarily requested sinus lift. All patients signed an informed consent for surgery and participation in scientific studies.

Patient inclusion criteria: age over 18 , no associated local or systemic disease, subantral bone available between 3-5 $\mathrm{mm}$, patient having signed an informed consent, patient in which the sinus membrane remained intact after elevation.

Under local anesthesia, the maxillary sinus was approached by opening a bone window in the lateral sinus wall. Subsequently, the sinus membrane was elevated, and the bone flap was mobilized in cranial position.

Under local anesthesia, an incision at the level of the vertical mandibular ramus was performed, which was extended to the lower vestibule. After the mandibular cortical bone was exposed, autologous bone material was taken from the vertical mandibular ramus and the external oblique line, and the postoperative wound at the donor site was sutured with separate non-resorbable threads (Fig. 1). The autologous material was milled using a manual bone mill and then, it was mixed in a 1/1 ratio with an alloplastic material consisting of PerioGlas particles (NovaBone poducts LLC), in a $0.9 \%$ saline solution medium. The sinus cavity was lined with the material thus obtained, and the bone window was covered with a resorbable collagen membrane and the mucoperiosteal flap. A non-resorbable separate stitches suture was performed.

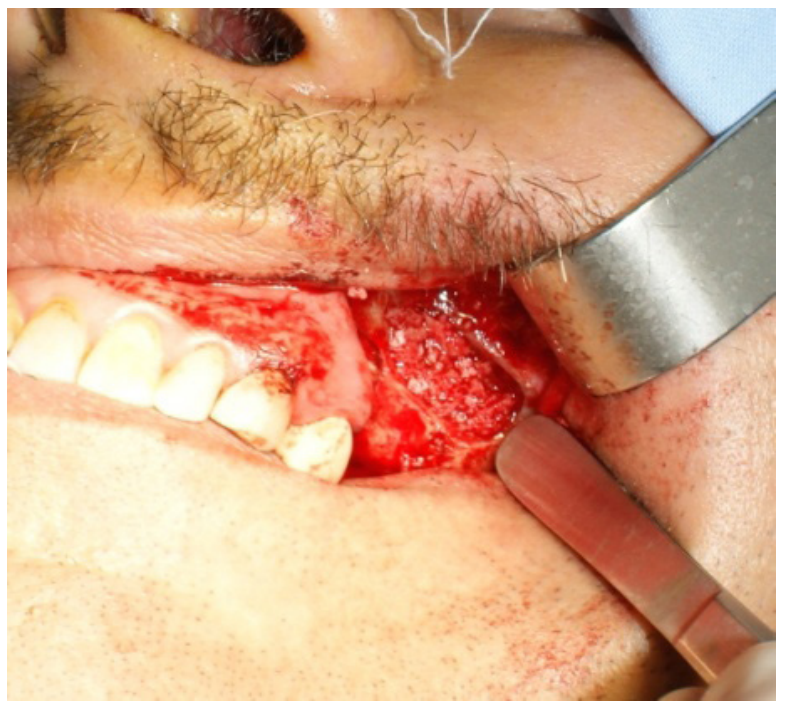

Figure 1. Placement of the augmentation material.

The suture was removed from the donor site at 7 postoperative days, and from the receptor site at 10 postoperative days. All patients received antibiotic treatment with amoxicillin with beta-lactamase inhibitors for 7 postoperative days, $2 \mathrm{~g}$ /day p.o., $1 \mathrm{~g}$ every 12 hours. Non-steroidal anti-inflammatory drugs were administered as needed.

A panoramic radiograph at a 1:1 scale was performed postoperatively, on which the obtained subantral bone level considered as a reference level was measured. At 6-8 months from bone augmentation, the patients returned for dental implant placement, and a new panoramic radiograph at a 1:1 scale was performed preoperatively, on which subantral bone height was measured. The difference between the initial and the final bone height represented the bone resorption rate of the augmentation material and was analyzed in nominal and percent terms.

During the bone implant placement procedures, bone tissue samples were taken from the detritus left on the drills used for the creation of new alveoli. These samples were preserved and sent for histological examination.

For histological analysis, the tissue samples were fixed in formaldehyde $4 \%$ and embedded in paraffin wax. Histological sections were cut at 5 micrometers and stained. Hematoxylin and eosin staining along with Masson's Trichrome, Sirius Red were used.

Data centralization, contingency tables and statistical interpretation were performed using the Microsoft Excel software. 


\section{Results}

Of the 7 patients included in the study, 3 (42.86\%) were males and 4 (57.14\%) were females. The age of the patients ranged between 42 and 68 years, with a mean age of 56 years. Following subantral bone augmentation, a bone height between 13 and $17 \mathrm{~mm}$, with a mean value of $15.14 \mathrm{~mm}$, was obtained, and 6-8 months after bone augmentation, values ranged between 14 and $16 \mathrm{~mm}$, with a mean height value of $12.71 \mathrm{~mm}$ (Fig. 2).

The resorption rate of the subantral augmentation material, as a nominal value, ranged between $1 \mathrm{~mm}$ and $3 \mathrm{~mm}$, with a mean resorption rate of $2.28 \mathrm{~mm}$. The resorption rate of the subantral augmentation material, as a percent value, ranged between $12.5 \%$ and $21.42 \%$, with a mean value of $15.15 \%$.

The histological analysis of the subantral augmentation material 6-8 months after bone augmentation (Fig. 3) shows an amorphous material percentage between $45.16 \%$ and $74.12 \%$, with a mean of $61.4 \%$.

The presence of an increased amorphous material amount is correlated with a lower resorption rate of the augmentation material and implicitly, with a smaller reduction of subantral bone height (Fig. 4).

\section{Discussion}

The objectives of the study were fulfilled. Thus, the subantral bone resorption rate was objectively analyzed at a reference interval relevant both for the scientific community and the medical community.

The presented data indicate a moderate resorption rate of the subantral augmentation material compared to other literature studies $[8,9,10]$. Thus, an analysis of the resorption rate of alloplastic grafts shows a lower resorption rate of these grafts compared to that reported in the present study [8]. This lower resorption rate of the alloplastic material used for subantral augmentation, compared to that found in patients with bone augmentation using a mixture of alloplastic and autologous material, is not surprising. In fact, the mixture of the two categories of materials is used in order to combine the advantages of each. The resorption rate of the alloplastic augmentation material is lower compared to that of the autologous material, and their association is aimed at slowing the resorption rate of the subantral graft [11]. On the other hand, autologous graft is considered to be the bone augmentation material with the best biological properties, so far unequaled by any other material [2], but previous studies indicate the increased bone resorption rate of this type of material as a disadvantage $[12,13]$. The position of the mean resorption rate obtained in this study between the rate of autologous grafts and that of alloplastic grafts is an expected result, which confirms the hypothesis that an association of the two types of materials improves their characteristics.

The qualitative analysis of the subantral augmentation material can only be performed through

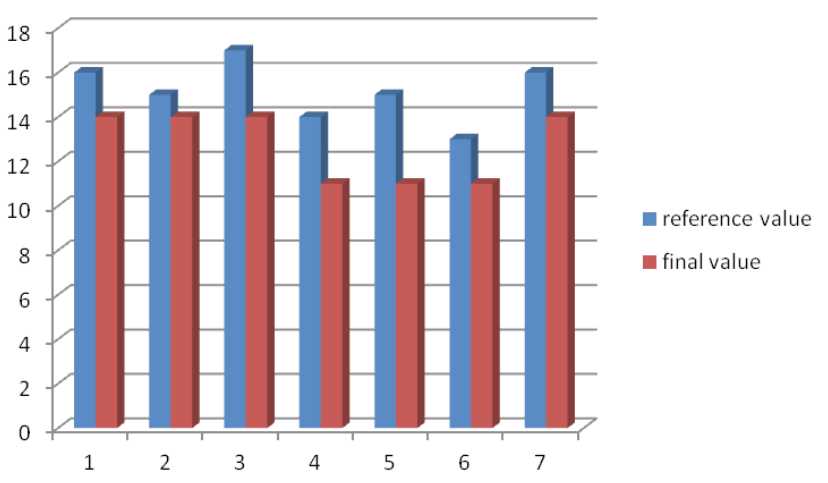

Figure 2. Values of subantral bone height immediately after bone augmentation and at the time of dental implant placement (6-8 months after bone augmentation).

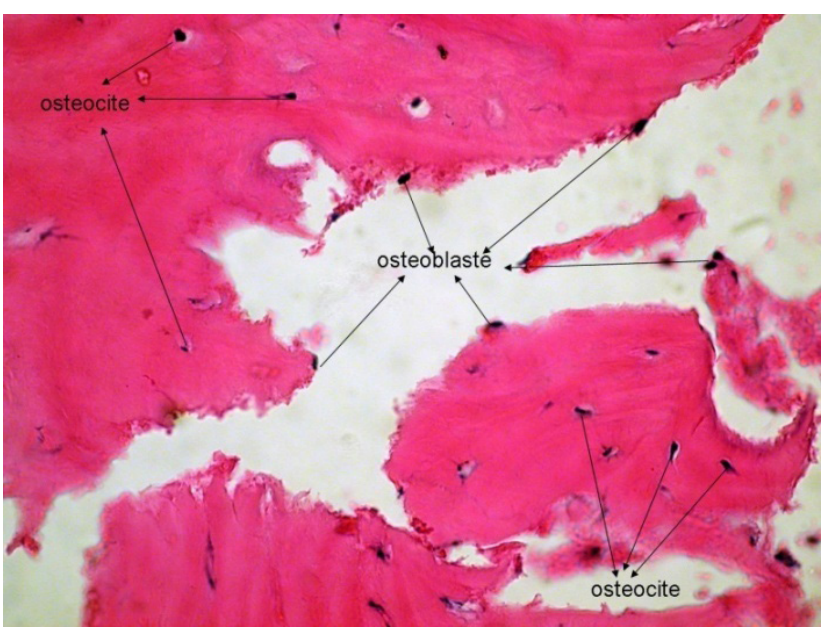

Figure 3. Autologous graft, HE staining 400X.

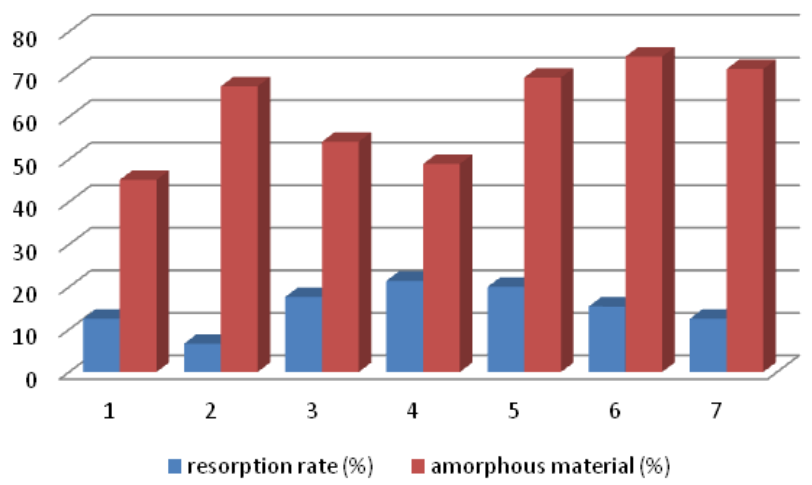

Figure 4. A comparative representation of the subantral bone resorption rate and of the residual amorphous material amount at the level of the bone graft.

histological studies. For ethical reasons, these studies can be performed on human subjects only when surgery for therapeutic purposes is necessary. Thus, the collection of biological samples for histological examination is limited to the stage of dental implant placement, when tissue debris results, which can be sent for histological analysis. The aim of combining alloplastic and autologous material 
is to slow the bone resorption rate of the grafts, which is characteristic of alloplastic grafts, and to stimulate neoosteogenesis, which is characteristic of autologous grafts. The histological examination performed after the collection of biological samples from the bone augmentation areas evidences varied amounts of amorphous material in the bone augmentation areas. On an average, amorphous material represents slightly more than half of the material volume present in the maxillary sinus. This percentage is higher than that detected in patients who underwent bone augmentation with autologous material alone $[4,9,14]$. On the other hand, in the case of bone augmentation with alloplastic grafts alone, there was a higher residual amorphous material amount in the augmentation area compared to that found in the patients of this study $[4,14]$. It should be mentioned that in the case of the studies analyzed for comparing the results obtained, the analysis of the subantral augmentation material was performed after a similar number of months from sinus lifting. Thus, it can be seen that the mixture of the two types of materials allowed to combine their characteristics, while their efficiency in obtaining dental implant osseointegration remains to be evaluated.

It should be noted that the presence of a higher amount of residual amorphous material in the bone augmentation area is associated with a lower resorption of the augmentation graft. Hence, the presence of intense osteogenic processes leads to an increase in the resorption rate of the subantral augmentation material. Further studies are required to establish to what extent the acceleration or slow-down of osteogenic phenomena affects the capacity of the bone augmentation area to receive and integrate dental implants.

\section{Conclusion}

The 1/1 mixture of alloplastic and autologous materials used for maxillary sinus augmentation leads to an improvement of the characteristics that each material has when used alone.

\section{Acknowledgement}

This article was published through the European Social Found, the Human Resources Development Operational Program 2007-2013, project no. POSDRU/159/1.5/S/138776

\section{References:}

1. Hieu PD, Chung JH, Yim SB, Hong KS. A radiographical study on the changes in height of grafting materials after sinus lift: a comparison between two types of xenogenic materials. J Periodontal Implant Sci. 2010;40:25-32.

2. Peng W, Kim IK, Cho HY, Pae SP, Jung BS, Cho HW, et al. Assessment of the autogenous bone graft for sinus elevation. J Korean Assoc Oral Maxillofac Surg. 2013;39:274-282.

3. Tatum H Jr. Maxillary and sinus implant reconstructions. Dent Clin North Am. 1986;30(2):207-229.

4. Bortoluzzi MC, Manfro R, Fabris V, Cecconello R, Derech ED. Comparative study of immediately inserted dental implants in sinus lift: 24 months of follow-up. Ann Maxillofac Surg. 2014;4(1):30-33.

5. Onişor-Gligor F, Rotaru A, Lung T, Juncar M, Mureșan O. Autologous and alloplastic sinus graft integration - comparative study. Journal of Romanian Medical Dentistry. 2010 14(1):60-64. 6. Dragoo MR, Sullivan HC. A clinical and histological evaluation of autogenous iliac bone grafts in humans. I: Wound healing 2 to 8 months. J Periodontol. 1973;44:599-613.

7. McAllister BS, Margolin MD, Cogan AG, Buck D, Hollinger JO, Lynch SE. Eighteen-month radiographic and histologic evaluation of sinus grafting with anorganic bovine bone in the chimpanzee. Int J Oral Maxillofac Implants. 1999;14:361-368.

8. Heinemann F, Mundt T, Biffar R, Gedrange T, Goetz W. A 3-year clinical and radiolographic study of implant placed simultaneously with maxillay sinus floor augmentations using a new nanocryztaline hydroxyapatite. J Physiol Pharmacol. 2009;60 (Suppl 8):91-97.

9. Xavier SP, Dias RR, Sehn FP, Kahn A, Chaushu L, Chaushu G. Maxillary sinus grafting with autograft vs. fresh frozen allograft: a split-mouth histomorphometric study. Clin Oral Implants Res. 2014 Apr 16. doi: 10.1111/clr.12404. [Epub ahead of print]

10. Kim ES, Moon SY, Kim SG, Park HC, Oh JS. Threedimensional volumetric analysis after sinus grafts. Implant Dent. 2013;22(2):170-174.

11. Nishibori M, Betts NJ, Salama H, Listgarten MA. Short-term healing of autogenous and allogeneic bone grafts after sinus augmentation: a report of 2 cases. J Periodontol. 1994;65:958966.

12. Pinchasov G, Juodzbalys G. Graft-free sinus augmentation procedure: a literature review. J Oral Maxillofac Res. 2014;5(1):e1. 13. Onişor-Gligor F, Rotaru A, Juncar M, Bran S. Studiu clinic privind integrarea grefelor sinuzale și a implanturilor în regiunea maxilară posterioară. Rev Med Chir Soc Med Nat Iaşi. 2009;113(4):1141-1145.

14. Sununliganon L, Peng L, Singhatanadgit W, Cheung LK. Osteogenic efficacy of bone marrow concentrate in rabbit maxillary sinus grafting. J Craniomaxillofac Surg. 2014;42(8):1753-1765. 